\title{
Presacral extramedullary hematopoiesis: a rare cause of back pain in a patient with thalassemia
}

\author{
Maroua Slouma $^{1}$ (D) Salma Athimni ${ }^{1} \cdot \operatorname{Rim}_{\text {Dhahri }}{ }^{1} \cdot$ Samy Zriba $^{2} \cdot$ Wajdi Amorri $^{3} \cdot$ Imen Gharsallah $^{1} \cdot$ Leila Metoui $^{1}$. \\ Bassem Louzir ${ }^{1}$
}

Received: 31 March 2020 / Revised: 23 June 2020 / Accepted: 1 July 2020 / Published online: 7 July 2020

(C) International League of Associations for Rheumatology (ILAR) 2020

\section{Presentation}

A 31-year-old man, with a history of $\beta$-thalassemia intermedia, presented with a 3-month history of low back and buttock pain. He underwent a splenectomy at the age of 9. He was under deferasirox treatment. Physical examination showed tenderness in the sacral area. Laboratory examinations showed anemia. The magnetic resonance imaging (MRI) revealed a well-defined presacral mass with hypo-intensity on T1- and T2-weighted images (Fig. 1a-c). The diagnosis of extramedullary hematopoiesis (EMH) was made based on the history of thalassemia and the imaging findings. He underwent conservative treatment. After 12 months of follow-up, MRI showed stability of this mass (Fig. 1d, e). The patient reported a $70 \%$ improvement in symptoms. Given the high risk of bleeding, he refused surgical excision.

Written informed consent for the case to be published was obtained from the patient.

\section{Discussion}

EMH is a benign proliferation of hematopoietic cells outside the bone marrow as a compensative process for the insufficient production of blood cells. It is due to the activation of

Maroua Slouma

maroua.slouma@gmail.com

1 Department of Internal Medicine, Military Hospital, Tunis El Manar University - Tunisia, 1007 Tunis, Tunisia

2 Department of Hematology, Military Hospital, Tunis El Manar University - Tunisia, Tunis, Tunisia

3 Department of Radiology, Military Hospital, Tunis El Manar University - Tunisia, Tunis, Tunisia primitive hematopoietic stem cells in fetal hematopoiesis sites $[1,2]$.

EMH mostly affected patients with an underlying hematological disorder. It occurs in about $20 \%$ of patients with thalassemia intermedia [2].

Only 14 cases of presacral EMH had been reported until 2009 [3]. It was related to thalassemia in 5 cases [3].

EMH is often asymptomatic. It can be responsible for bowel and bladder symptoms. Among these 14 patients, low back pain and sciatica were reported in one case each [3].

Differential diagnoses of presacral mass include myelolipomas, well-differentiated liposarcoma, and fibrosarcoma [4].

Presacral EMH is a well-defined round or macro-lobulated presacral mass. The MRI signal depends on the activity of the lesion. Indeed, active lesions show iso- or hypo-intensity on both T1- and T2-weighted images [4, 5]. However, older inactive lesions show hyper-intensity on $\mathrm{T} 1$ and $\mathrm{T} 2$ sequences, highlighting the fatty infiltration [5]. Gadolinium enhancement is variable [5].

Myelolipomas are often a well-demarcated and heterogeneous tumor with nodular soft tissue and fatty components. Liposarcoma is a fat-containing tumor, rarely confined to the presacral space.

The presacral mass may also originate from sacrum with anterior extensions such as giant cell tumors, chordomas, osteosarcoma, and chondrosarcoma. In these cases, imaging showed osseous destruction.

It may be due also to metastasis from primary pelvic malignancy, lymphoma, or teratoma.

Regarding presacral lymphoma, MRI shows hypointensity on T1 and hyper-intensity on T2-weighted images. The identification of surrounding or distant lymph nodes helps to make the diagnosis of lymphoma.

Histological findings provide better diagnostic accuracy. However, given the high risk of bleeding, histological confirmation is not always necessary. Indeed, among the 14 patients 


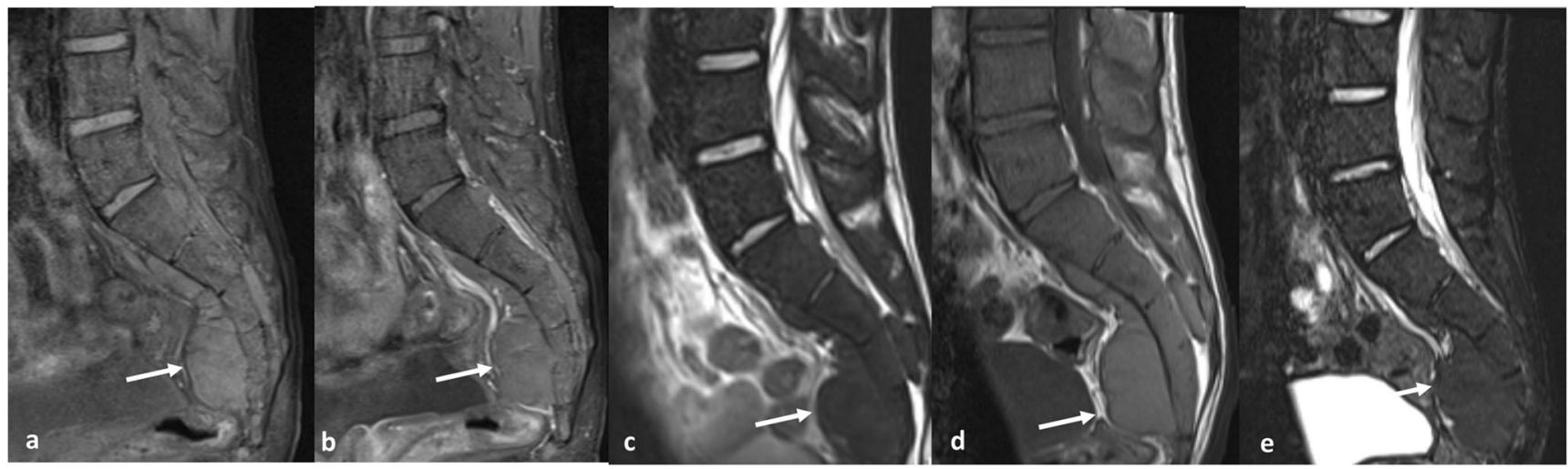

Fig. 1 Sagittal MRI of the sacrum showing a well-defined presacral mass (white arrow), measuring $80 \times 25 \mathrm{~mm}$, adjacent to the sacral vertebrae with slight hypo-intensity on T1-weighted image (a), a low level of homogeneous enhancement after the intravenous injection of gadolinium (b), and hypo-intensity on T2-weighted image (c). There was no bone destruction. MRI after 1 year of follow-up showing the stability of this presacral mass on T1-weighted image after the intravenous injection of gadolinium (d) and T2-weighted image (e) with presacral EMH, a biopsy was performed in 7 cases [3]. Characteristic MRI findings in patients with thalassemia are sufficient to make the diagnosis of EMH [5].

The management of presacral EMH may include conservative treatment, radiotherapy, and regular blood transfusions [1, 3]. Surgical excision is associated with the risk of bleeding and recurrence of the lesion [3].

There are no recommendations regarding follow-up imaging. The authors suggest that the follow-up MRI is required to verify the stability of the lesion [4]. It was mostly performed 6 to 12 months after the diagnosis $[3,5]$.

Presacral EMH is a rare etiology of low back pain in a patient with thalassemia. We highlighted the importance of MRI findings, which depend on the activity of the lesion. This diagnosis should be kept in mind to avoid expensive and unnecessary explorations.

\section{Compliance with ethical standards}

Disclosures None.

\section{References}

1. Yang X, Chen D, Long H, Zhu B (2020) The mechanisms of pathological extramedullary hematopoiesis in diseases. Cell Mol life Sci . Advance online publication. https://doi.org/10.1007/s00018-02003450-w

2. Rajiah P, Hayashi R, Bauer TW, Sundaram M (2011) Extramedullary hematopoiesis in unusual locations in hematologically compromised and noncompromised patients. Skelet Radiol 40(7):947-953. https://doi.org/10.1007/s00256-011$1129-\mathrm{z}$

3. Babazadeh S, Broadhead ML, Slavin JL, Choong PF (2009) An interesting diagnosis for a presacral mass: case report. Int Semin Surg Oncol 6:18. https://doi.org/10.1186/1477-7800-6-18

4. Sohawon D, Lau KK, Lau T, Bowden DK (2012) A pictorial review of its typical and atypical locations: extramedullary haematopoiesis locations. J Med Imaging Radiat Oncol 56(5):538-544. https://doi. org/10.1111/j.1754-9485.2012.02397.x

5. Wong KH, Li A, Lui TH, Sit YK (2014) Spinal epidural extramedullary haematopoiesis in $\beta$-thalassaemia intermedia. BMJ Case Rep:bcr2013201534. https://doi.org/10.1136/bcr-2013201534

Publisher's note Springer Nature remains neutral with regard to jurisdictional claims in published maps and institutional affiliations. 